\title{
Personalized Medicine in Rare Diseases
}

\section{Francesc Palau}

Instituto de Biomedicina de Valencia, CSIC, and CIBER de Enfermedades Raras (CIBERER), Valencia, Spain

Address correspondence:

Francesc Palau, MD, PhD

Instituto de Biomedicina de Valencia, CSIC

C/ Jaume Roig, 11

46010 Valencia, Spain

Phone: $+34-96-339-3773$

Fax: +34-96-369-0800

e-mail: fpalau@ibv.csic.es 
Definition of personalized medicine is not an easy issue $[1-3,101]$. It is moving from personalized medical practice to the modern concept of personalizing the science of medicine to improve individual healthcare. In many aspects personalized medicine refers to translation into clinical medicine and health care person's unique biological individuality and his/her interaction with the environment. By the term personalized medicine we envision a complete integration of clinical (phenotype), genetic, genomic, transcriptomic, proteomic, metabolomic profiles with environmental (including nutritional) information that is provided for a particular person. The major expected consequence is to optimize preventive health care strategies and response to drug therapies while people are either healthy or in an early stage of the disease. Personalized medicine tries to move the population-based evidence of medical interventions towards individual evidence of how to treat the specific person based on the biological profile, clinical history and environment. The aim is to offer a tailored healthcare to every person. The personalized medicine based on the integration of individual information, from the genome variation, physiology and cellular phenotype to the interaction with the personal environment, may represent a proactive, preventive and prospective model of health care [4-6] by opposite to the more traditional, diseasebased, reactive approach to the health status of individuals.

The fundamentals of genomic and personalized medicine and application of new technologies that may define personal biological profiles apply to every type of pathological process, disease or condition, whatever the main organ or tissue is involved in the disorder affecting the person. Personalized medicine today is addressing a number of elements that are relevant to any kind of disease. Such elements have to be considered when we are thinking in a prospective model of health care and generation of predictive markers. They include basic research and technologies development (most of them based on 'omics' approaches), translational research and characterization of biomarkers, further clinical application of new 
translational tools, and social and ethical impact. However, the impact of personalized medicine has not been identical in all clinical areas. Traditionally, personalized medicine has been in practice in oncology and across other disease specialisms involving common diseases such as cardiovascular disorders, diabetes and metabolic syndrome, and neurodegenerative diseases. In addition, in the recent years rare diseases (RD) have become an important field of interest for both translational research and the application of personalized medicine approach to individual patients. How personalized medicine may be incorporated in the health care of RD? Are the premises and fundamentals identical among RD to the common diseases (CD)? Which are the main differences among $\mathrm{RD}$ and $\mathrm{CD}$ for the individual application of personalized medicine? To address these questions we need to answer some elements of medical thinking by responses that characterize RD.

\section{Elements for a conceptual framework of rare diseases}

By 'rare diseases' we refer to non-frequent disorders or conditions with a low prevalence that in the European Union has been estimated of less than 5 patients per 10,000 inhabitants. According to that definition, RD affect $6-8 \%$ of the Europe's population, that is, around 30 million people in the EU-27 are affected. Despite their low frequency these diseases show some common characteristics, which allow integrating individuals affected by a RD as a social group that share common health and social problems $[7,102,103]$. There are important factors to take in account when thinking in a RD: (i) they used to be severe, chronic and progressive disorders with a high degree of incapacity; (ii) in spite of they are not common disorders RD are complex pathological conditions, and (iii) in most cases, RD are genetic disorders that express the first symptoms at the pediatric age. 
The individual patient and also patients as a whole increasingly own their life and their ability to make decisions, with appropriate information and autonomy; in some way it can be stated that they are personalized decisions. The empowerment that patients have on their own illness, based on their experience and knowledge of life, makes them fundamental for the management of the disease and the lifestyle according to their needs. No matter how we define the RD, patients have much to say about them. Some important issues that affect patients and for which they and the lay organizations are demanding attention may be summarized as follows [103]: lack of scientific knowledge about their illness, difficulty of access to correct diagnosis, delayed diagnosis, lack of care and multidisciplinary care, lack of quality information and support at the time of diagnosis, adverse social consequences, difficulty and lack of equity in access to treatment, rehabilitation and care, loss of confidence in medical and social services and a lack of interest from health professionals.

As mentioned above RD are a broad and heterogeneous group of severe and disabling disorders, involving a small number of individuals in specific populations. The disease course used to be chronic with high morbidity and mortality, and therapies are scarce. However, there are common diseases showing a chronic and disabling natural history, with high morbidity and mortality rate, and limited therapeutic arsenal. Thus, what does make the difference among rare diseases and common chronic diseases? In the following paragraphs I propose several elements that could help to create a single framework of the aspects that characterise rare diseases [8, 104]. These elements are important when applying personalized medicine concepts and technologies to a person affected by a RD:

Public health component. Each rare disease has a low prevalence, however, the involvement of roughly $30,000,000$ people with shared health problems in a population as the European Union, requires a scientific approach based on public health criteria and premises. This is what has been called the paradox of rarity. 
Natural history: diversity and heterogeneity. In a set of clinical conditions as wide as RD diversity is an intrinsic feature. The nature of diseases ranges from diseases that affect a single organ system until illnesses that are multisystemic. The heterogeneity of these diseases is manifested in different profiles of natural history, including the age at onset, which determines the clinical intervention and preventive health services.

Multidisciplinary and comprehensive healthcare. A RD is often chronic and disabling. In many cases patients with these diseases need care that goes beyond the specific clinical care offered by a unique specialized service. This care should be considered in the context of overall management, with comprehensive medical and psychological care.

Social and health integrative care. Many RD involve a high degree of dependency and social, health and economic burden. This is not something particular to the RD, but there are other aspects that are specific to them and give them a certain degree of particularity. As previously mentioned, one of these specificities is the age at onset of the disease. Most of RD used to express first symptoms early in the biography of patients in the pediatric period of life, sometimes between birth and young adult life, being early onset the most frequent situation. Thus, in many cases patients require health and social care for a very long period of their lives.

Genetic inheritance. Eighty percent of the RD is estimated to be genetic, mostly monogenic, and follow the laws of Mendelian inheritance (also mitochondrial inheritance). Knowledge about the genetic causes -mutations- and the underlying pathomechanisms are very important to address further research and translation into clinical medicine of these diseases.

Risk of recurrence. Risk of recurrence of a genetic disease on the families makes genetic counseling a support and therapeutic tool for these patients and their families, which is fundamental for planning reproductive decisions, prenatal diagnosis or preimplantational genetic diagnosis. 
Geographic dispersion. Except in cases of adverse toxicological, infectious agents or other environmental causative factors that may affect a specific population, disease used to be present in any population. This is especially true for chronic disorders either common or rare. However, the association of both random distribution and low prevalence observed for $\mathrm{RD}$ is a phenomenon intrinsic to these disorders. This circumstance induces the isolation of the patient and the family with no contact with other affected people. At the beginning after diagnosis patients or parents may feel that they are alone as they never have listen to that disease. Even when they know about other patients is very frequent that no more individuals are living close to them.

Treatment opportunities and development of therapies. Therapeutic options in RD are generally scarce and ineffective. However, the development of new therapies and drugs for these diseases aimed at specific molecular targets of RD may be useful for not only themselves but also for common diseases that may share such biological targets or pathophysiogical pathways.

\section{Models of medicine and healthcare}

The $20^{\text {th }}$ century introduced modern scientific and experimental knowledge into medicine and clinical practice. Even more important, European and other countries have developed national health systems to provide care to the population. Childs distinguishes two point of view of disease [9]. The Osler's view of patient as a broken machine: historically, in most cases the clinical approach to the patient is based on this essentialist view of disease, that is, the patient is represented in the "classical case" and the physician is treating the disease but not the individual patient. By contrast, the view of Garrod is based on the individuality of the patient: there are no disease, only sick people. The individuality (chemical) is that of the patient who represents his or her 
own self. However, for both points of view of disease current model in health care is still reactive: intervention begins once disease is established either as acute onset or acute episodes in chronic diseases. In opposition to the reactive model, even when considering the individuality of the patient, Snyderman and colleagues [4-6] propose a model of health care based on "the concept of strategic health planning, a nonreductionist, proactive, prospective approach to care". In that system, the relevance for the individual's health underlays on the patient's baseline risk for various diseases (familial history may be relevant), his or her health status, and the likelihood of developing specific clinical problems regarding the estimated risks. Disease is the consequence of multiple factors including genetic susceptibilities and environmental exposure (baseline risk). Over time the disease appears and progress from the baseline to irreversibility. Specific actions on the individual may help to predict and to prevent risks on developing the disease. An outcome of the prospective model is disease prediction. In this model demographic, gender, clinical, and family-history predictors will probably retain their value. But these are not enough. We need to recognize new biomarkers and to define their role on the prognosis of disease and response to therapies, and even more relevant to their role on the prediction and anticipation of disease. One of the major drivers to discovery predictive factors and development of personalized medicine is genomic research and genomic application to clinical medicine and health care. Stable gene variation and genome predictors will increase baseline clinical risk assessment and the need for primary prevention of a particular individual, whereas dynamic factors that show variation over time such as mRNA, proteins and metabolites will enhance refined risk assessment giving the opportunity to act on the disease progression and guide therapeutic options. Has this prospective model identical application to RD than CD? Are there any differences on baseline risks and prediction for $\mathrm{RD}$, especially single-gene disorders? 


\section{A working comparison among rare diseases and common diseases}

To address this point we are comparing the above-mentioned elements of medical thinking for both RD and CD. First of all, we have to be aware that boundaries among $\mathrm{RD}$ and $\mathrm{CD}$ are not perfectly established; thus, the adhesion to the criteria is always relative and not absolute. The elements appear in Table 1, which contrasts characteristics of both types of diseases, separated not only by the criterion of frequency but also by the consequences of such a relative prevalence in the populations:

- Most of RD, if any, does not represent by itself a major health problem. However, $\mathrm{RD}$ as a whole require an approach using the tools of public health as most of specific common diseases.

- Again, most of the RD used to have an early age at onset in infancy, childhood, adolescence or early adulthood; by contrast, most of CD used to onset in the adulthood or elderly time. Thus, the whole biography of the patient may be affected by the disease.

- It is possible to state that in many cases of RD the individual life has not been free of disease. This aspect also involves the need for an integrated social and health care programme for a long period of the patient's life.

- Almost every disease is related to the genetic background of the individual and the interaction between the genomic profile and environmental forces. However, rare genetic diseases are single-gene disorders. For the affected individual this circumstance represents the baseline to develop the disease. In such a case to be carrier of one or two pathogenic mutations almost fully correlated to the appearance of the disease. On the contrary, the genetic susceptibility to develop a common disease is based on the presence of a number of frequent and/or rare genomic variants [10]. 
- In the previous context, the risk of recurrence of the disease within the family is much higher for RD than for CD.

- Patients for a specific disease are dispersed and there are not any a priori reason to expect for it in any population (toxic, infectious diseases or the effect of neck bottle of a monogenic disorder may be excluded). But patients affected by a RD are more isolated as, at least at diagnosis, they do not know about other person affected by the same disease.

- The interest for drug or therapy development by pharmaceutical companies is opposite when comparing $\mathrm{RD}$ and $\mathrm{CD}$. The orphan drugs policy in the European Union and USA has changed the future of the investment on therapy research on $\mathrm{RD}$; in addition, therapeutics research on $\mathrm{RD}$ may be the opportunity to new solutions not just for the specific RD but also for common diseases that may have some pathophysiological connexions with the rare disease.

\section{How we understand personalized medicine in rare diseases}

Based on the elements described above we have showed several aspects that may differentiate $R D$ and $C D$. In fact, differences on the age at onset are very important. However, most of the biological and clinical elements related to personalized medicine and the prospective model of disease [4-6] are shared by RD and CD. Identification of genomic and other omics profiles in every individual either affected or healthy will be relevant to design his/her care. Thus, are there any the specific aspect of personalized medicine in rare diseases? Part of the answer is already mentioned previously, but another one is related to the development of biomarkers (Table 2): whereas common diseases may have biological diagnosis (diagnostic biomarkers), few biomarkers are available diagnosis of rare diseases. Static genomic biomarkers are relevant for $C D$ as they are defining the susceptibility for a trait or disease; the combination of such 
genomic variants may increase or decrease the baseline of the disease in the individuals of a population. By contrast, the baseline is very low in RD patients with a highly penetrant mutation. Dynamic biomarkers to define the prognosis and therapeutic response for both $C D$ and $\mathrm{RD}$ are also needed. In both cases biomarkers should be related to the biological pathways involved in the disease process. Again, a major difference among $\mathrm{RD}$ and $\mathrm{CD}$ is the pivotal role the pathogenic primary mutation in the origin and further evolution of the disease.

\section{References}

1. Ginsburg GS, Willard HF. The foundations of genomic and personalized medicine. In: Essentials of Genomic and Personalized Medicine. Ginsburg GS, Willard HF (Ed.). Academic Press, Elsevier Inc., San Diego, USA, 1-10 (2010).

2. Ginsburg GS. Translational genomics: from discovery to clinical practice. In: Genomic and Personalized Medicine (Volume 1). Willard HF and Ginsburg GD (Ed.). Academic Press, Elsevier Inc., San Diego, USA, 261-274 (2009).

3. Hamburg MA, Collins FS. The path to personalized medicine. $N$ Engl J Med 363: 301-304 (2010).

4. Snyderman R, Yoediono Z. Prospective care: a personalized preventive approach to medicine. Pharmacogenomics 7: 5-9 (2006).

5. Snyderman R, Langheier J. Prospective health care: the second transformation of medicine. Genome Biol 7: 104 (2006).

6. Snyderman R. The role of genomics in enabling prospective health care. In: Genomic and Personalized Medicine (Volume 1). Willard HF and Ginsburg GD (Ed.). Academic Press, Elsevier Inc., San Diego, USA, 378-385 (2009). 
7. Schieppati A, Henter JL, Aperia A. Why rare diseases are an important medical and social issue. Lancet 371, 2039-2041 (2008).

8. Palau F. Enfermedades raras, un paradigma emergente en la medicina del siglo XXI. Med Clin [Barc] 134: 161-168 (2010).

9. Childs B. Genetic Medicine - A Logic of Disease. The Johns Hopkins University Press, Baltimore \& London, (1999).

10. Manolio TA, Collins FS, Cox NJ et al. Finding missing heritability of complex diseases. Nature 461: 747-753 (2009).

\section{Electronic references}

101. Personalised Medicine for the European citizen - towards more precise medicine for the diagnosis, treatment and prevention of disease. URL:

http://www.esf.org/activities/forward-looks/medical-sciences-emrc/current-forwardlooks-in-medical-sciences/personalised-medicine-for-the-european-citizen/moreinformation.html

102. Rare diseases: understanding this public health priority. URL:

http://www.eurordis.org//MG/pdf/princeps document-EN.pdf

103. The voice of 12,000 patients. URL:

http://www.eurordis.org/article.php3?id article=1960

104. Palau F. Introduction. In: Rare Diseases Strategy of the Spanish National Health System, Ministry of Health and Social Policy, Madrid, Spain, 15-20 (2009)

http://ciberer.es/documentos/Rare\%20Diseases\%20Strategy\%20of\%20the\%20Spanis h\%20National\%20Health\%20System.pdf 


\section{Acknowledgements}

Dr. Palau's laboratory is funded by grants from the Spanish Ministry of Science and Innovation, the Instituto de Salud Carlos III, the Frame Programme 7 of the European Comission, the Generalitat Valenciana and the Fundació La Marató TV3. 


\section{Table 1 Comparison of medical thinking elements}

\begin{tabular}{|c|c|c|}
\hline ELEMENTS & RARE DISEASES & COMMON DISEASES \\
\hline $\begin{array}{l}\text { The public health } \\
\text { component }\end{array}$ & $\begin{array}{l}\text { As a whole, RD are a problem of public } \\
\text { health }\end{array}$ & $\begin{array}{l}\text { Every common disease is considered a } \\
\text { problem of public health }\end{array}$ \\
\hline $\begin{array}{l}\text { Natural history - } \\
\text { age at onset }\end{array}$ & $\begin{array}{l}\text { Early onset for most of RD have: } \\
\text { infancy, childhood, adolescence, early } \\
\text { adulthood }\end{array}$ & Late onset in adulthood or elderly age \\
\hline Health care & $\begin{array}{l}\text { Integrative and multidisciplinary model } \\
\text { but common services shared by } \\
\text { distinct RD }\end{array}$ & $\begin{array}{l}\text { Integrative and multidisciplinary model } \\
\text { but different approach for each CD }\end{array}$ \\
\hline $\begin{array}{l}\text { Integrated social } \\
\text { and health care }\end{array}$ & $\begin{array}{l}\text { High disease burden because early } \\
\text { onset (the whole biography of the } \\
\text { patient may be affected) }\end{array}$ & $\begin{array}{l}\text { High disease burden because the large } \\
\text { number of patients }\end{array}$ \\
\hline $\begin{array}{l}\text { Genetic } \\
\text { inheritance }\end{array}$ & $\begin{array}{l}\text { In most cases, single-gene inheritance, } \\
\text { either Mendelian or mitochondrial }\end{array}$ & $\begin{array}{l}\text { Consequence of genetic and } \\
\text { environmental interaction } \\
\text { (complex/multifactorial inheritance)- } \\
\text { Genetic susceptibility to disease }\end{array}$ \\
\hline Family risk & $\begin{array}{l}\text { High risk of recurrence within the } \\
\text { family }\end{array}$ & $\begin{array}{l}\text { Low risk of recurrence within the } \\
\text { family }\end{array}$ \\
\hline $\begin{array}{l}\text { Geographical } \\
\text { distribution }\end{array}$ & $\begin{array}{l}\text { Patients are dispersed and they may } \\
\text { feel unique in their own place (except } \\
\text { in isolated populations) }\end{array}$ & Frequent in most populations \\
\hline $\begin{array}{l}\text { Treatment and } \\
\text { therapies }\end{array}$ & $\begin{array}{l}\text { Historically low interest for } \\
\text { pharmaceutical companies - } \\
\text { Opportunities for orphan medicines }\end{array}$ & High interest for pharmaceuticals \\
\hline
\end{tabular}


Table 2 Differences of biomarkers in rare and common diseases MONOGENIC RARE DISEASES COMMON DISEASES

The major static (genomic) biomarker is the Genomic biomarkers are related to disease primary pathogenic mutation susceptibility

Other genetic/genomic variants may be gene Genetic/genomic variants may represent the modifiers of the phenotype expression basis for oligogenic or polygenic inheritance of complex traits

Prediction in mainly based on primary mutations and affects family members. Prediction is based on dynamic biomarkers as Dynamic (physiological) biomarkers are prognosis and therapy response are as well important for prognosis and therapy response 